\title{
Familial imbalance in 16p13.11 leads to a dosage compensation rearrangement in an unaffected carrier
}

\author{
Alicia Delicado 1,2, Luis Fernández ${ }^{1,2 *}$, María Luisa de Torres ${ }^{1,2}$, Julián Nevado 1,2, Fe Amalia García-Santiago ${ }^{1,2}$, \\ Roberto Rodríguez ${ }^{3}$, Elena Mansilla1,2, María Palomares ${ }^{1,2}$, Fernando Santos-Simarro ${ }^{1,2}$, Elena Vallespín ${ }^{1,2}$, \\ María Ángeles Mori ${ }^{1,2}$ and Pablo Lapunzina ${ }^{1,2}$
}

\begin{abstract}
Background: We and others have previously reported that familial cytogenetic studies in apparently de novo genomic imbalances may reveal complex or uncommon inheritance mechanisms.

Methods: A familial, combined genomic and cytogenetic approach was systematically applied to the parents of all patients with unbalanced genome copy number changes.

Results: Discordant array-CGH and FISH results in the mother of a child with a prenatally detected 16p13.11 interstitial microduplication disclosed a balanced uncommon rearrangement in this chromosomal region. Further dosage and haplotype familial studies revealed that both the maternal grandfather and uncle had also the same 16p duplication as the proband. Genomic compensation observed in the mother probably occurred as a consequence of interchromosomal postzygotic nonallelic homologous recombination.
\end{abstract}

Conclusions: We emphasize that such a dualistic strategy is essential for the full characterization of genomic rearrangements as well as for appropriate genetic counseling.

Keywords: 16p13.11 duplication, Gene dosage compensation, Homologous balanced rearrangement, Mitotic NAHR

\section{Background}

The combination of array-based comparative genomic hybridization (aCGH) technology and subsequent parental fluorescence in situ hybridization (FISH) analysis looking for balanced parental rearrangements has revealed complex or unusual genomic rearrangements that seem more common than previously considered [1-3]. Recently, it has been also demonstrated that parental submicroscopic insertional translocations (ITs) underlie $\sim 2.1 \%$ of the apparently de novo interstitial pathogenic copy number variations (CNVs) [4].

At the same time, the clinical use of aCGH has lead to the detection of many CNVs, a number of them of uncertain clinical relevance. Patients with 16p13.11

\footnotetext{
* Correspondence: Ifernandezg@salud.madrid.org

${ }^{1}$ Instituto de Genética Médica y Molecular (INGEMM), IdiPAZ, Hospital Universitario La Paz, Paseo de la Castellana 261, 28046 Madrid, Spain ${ }^{2}$ CIBER de Enfermedades Raras (CIBERER), ISCIII, Madrid, Spain

Full list of author information is available at the end of the article
}

duplication and its reciprocal deletion have been previously reported and are relatively common. However, whereas the 16 p13.11 deletion showed to be a pathogenic CNV, the duplication was initially thought to be benign [5]. Other authors described an incomplete penetrance and/or variable expressivity, the same duplication being found both in mildly affected and unaffected relatives within the same family [6,7]. Clinical features such as cognitive impairment, behavioral disorders, congenital heart defects, skeletal malformations and abnormal magnetic resonance imaging (MRI) findings have been associated with $\mathrm{CNVs}$ involving 16p13.11 [8].

In this report we describe a child with a brain malformation detected by prenatal ultrasound in which a prenatal aCGH study identified a $1.78 \mathrm{Mb}$ duplication at 16p13.11. Further parental FISH analysis showed that the phenotypically normal mother was carrying an unusual apparently balanced rearrangement between the two chromosome 
16 homologs. An elder brother of the patient, and both the patient's maternal grandfather and maternal uncle, all three apparently phenotypically normal, were also carriers of the same $16 \mathrm{p}$ duplication detected in the proband.

\section{Methods \\ Clinical report}

Samples from the proband and his family were obtained after informed consent. Ethical approval was obtained for this study from the IRB at Hospital Universitario La Paz in Madrid (HULP-CEIC-PI1207). Research was performed in compliance with the Declaration of Helsinki.

The patient, a male, was the product of the third pregnancy of a 34 year old woman. Both parents were healthy, phenotypically normal and nonconsanguineous. The first pregnancy ended in an elective termination and the second resulted in a healthy boy. A prenatal ultrasound at 28 weeks gestation revealed agenesis of corpus callosum. Further examination at 37 weeks showed two interhemispheric cysts along with corpus callosum agenesis and colpocephaly. He was born at 40 weeks of gestation by spontaneous vaginal delivery. At birth, weight was 3660 g (75-90th centile), length $52 \mathrm{~cm}$ (90th centile) and head circumference $36 \mathrm{~cm}$ (90th centile). Perinatal period was uneventful and Apgar scores were 9 and 10 at 5 and 10 minutes, respectively. Renal ultrasound showed mild bilateral pelvicalyceal dilatation.

Although initial marsupialisation of the cysts carried out at 20 days of life showed some reduction in their volume, subsequent increase in their size despite multiple drainage interventions mandated the placement of a permanent ventriculoperitoneal shunt at 10 months of age.

He was last seen in clinic aged 14 months, the brain MRI showing a $50 \%$ reduction in cysts volume. At that time, his developmental evaluation disclosed a normal cognitive profile for age with a motor delay secondary to left side hemiparesis.

His older brother was assessed in clinic aged 3, showing at that time a normal phenotype, normal growth parameters and a normal development for his age. The maternal uncle and grandparents were not directly evaluated in the genetics clinic, but were referred as normal by other family members.

\section{Genomic and cytogenetic studies}

According to our workflow in prenatal samples with two or more ecographic markers, aCGH was performed in the patient and his parents, using a custom oligonucleotide array (KaryoArray ${ }^{\circ}$ v3.0, $8 \times 60 \mathrm{~K}$, Agilent Technologies, Santa Clara, CA) as described previously [9]. This array has an average density of one probe per $9 \mathrm{~Kb}$ in clinically relevant regions (microdeletion/microduplication syndromes, subtelomeric and pericentromeric regions) and one probe per $175 \mathrm{~Kb}$ in other genomic regions including 16p13.11 (backbone). Further versions of this array will include this and other regions as they are associated with clinical phenotypes.

Karyotyping and FISH were performed on cultured peripheral blood lymphocytes. FISH studies were performed using standard procedures applying probe NDE1-MYH11 mapping 16p13.11 (Agilent Technologies, Inc., Santa Clara, CA).

Short tandem repeat (STR) markers segregation studies were carried out in the family to confirm gene dosage and to determine the parental origin of the rearranged chromosomal material. Genomic DNA was obtained using standard procedures from peripheral blood lymphocytes in all family members (QIAGEN, Valencia, CA), and from buccal swab in the mother (DNA Genotek, Kanata, Canada). Three polymorphic markers mapping to region 16p13.11 (D16S3060: 15,860,105-15,860,300; D16S3127: 15,870,885-15871004; D16S405: 15,882,592-15,882,733; hg19) and other nine markers located along chromosome 16 (D16S3070, D16S3088, D16S3024, D16S3079, D16S3114, D16S500, D16S690, D16S753, D16S3043) were analyzed.

Further studies to determine the presence of the gene dosage alteration in other family members were also carried out by Multiplex Ligation-dependent Probe Amplification (MLPA) using SALSA P092-B3 probemix containing probes for genes $A B C C 6$ and $A B C C 1$ in 16p13.11 (MRC-Holland, Amsterdam, The Netherlands).

\section{Results}

Array comparative genomic hybridization in the patient revealed a gain of $1.78 \mathrm{Mb}$ on 16p12.3-p13.11 (hg19, chromosome 16: 15,111,247-16,895,894) (Figure 1a). Genes included in this region are: PDXDC1, NTAN1, RRN3, MPV17L, C16orf45, KIAA0430, MIR484, NDE1 (MIM 609449), MYH11 (MIM 160745), C16orf63, ABCC1, ABCC6 (MIM 603234), NOMO3, PKD1P1. This finding was interpreted as a $\mathrm{CNV}$ of uncertain significance since this region had been reported to show copy number variation in individuals with no obvious phenotype, it contained several MIM genes and there was an ongoing debate as to the exact clinical significance of the CNV. Further aCGH studies on both parents were normal (Figure 1d) thus the duplication was initially considered an apparently de novo event in the boy.

Duplication in the proband could have hardly been detected in metaphase FISH studies, although retrospective re-evaluation of images noted a brighter signal pattern on one homologue (Figure 1b). Further interphase nuclei analysis with the same probe demonstrated actually three signals, two of them located in close proximity (Figure 1c). These findings supported the results of 


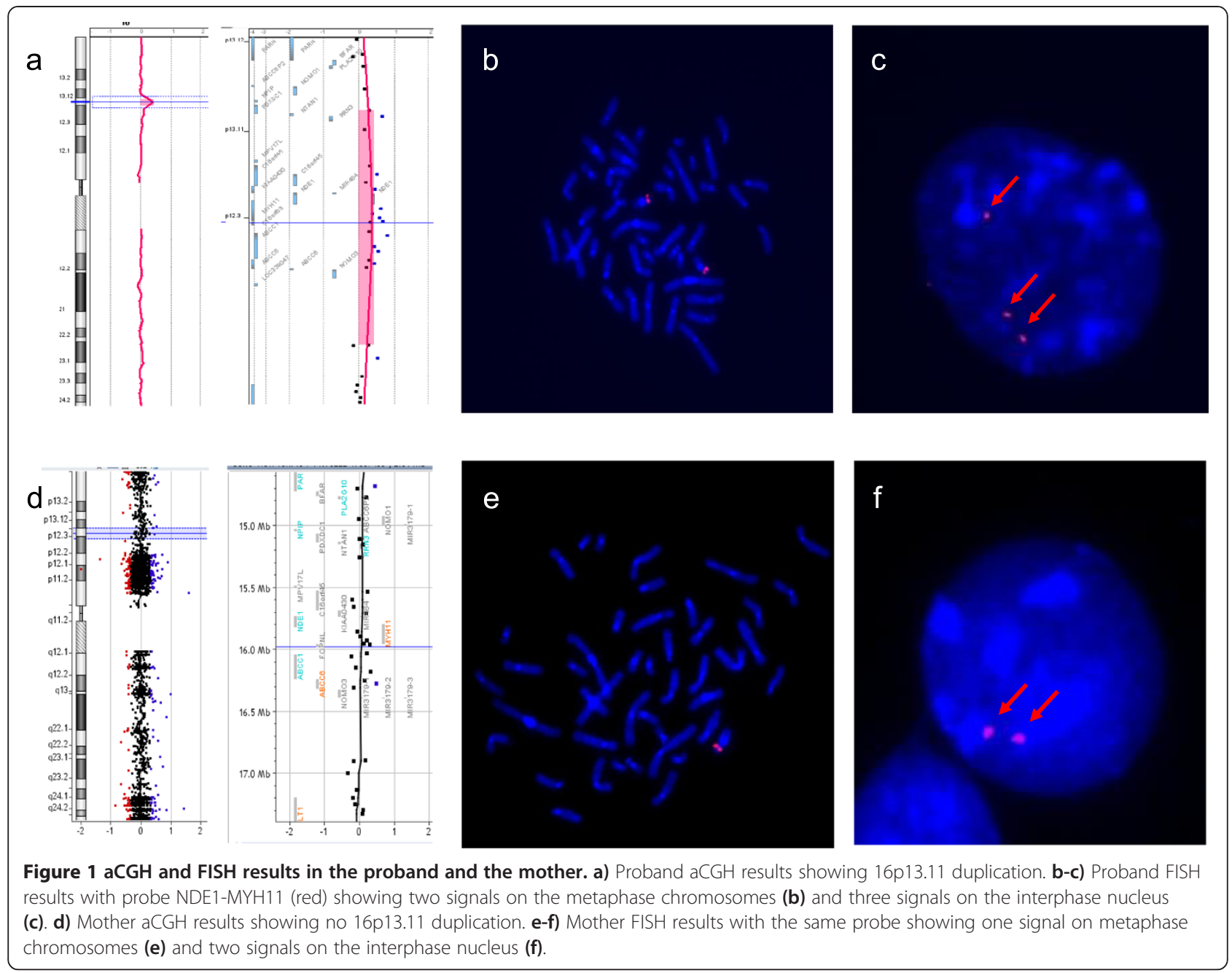

aCGH and allowed us to establish that the gene dosage amplification observed in the patient was located within that same region. His karyotype was then described as: ish $\operatorname{dup}(16)(\mathrm{p} 12.3 \mathrm{p} 13.11)(\mathrm{NDE} 1-\mathrm{MYH11}$ enh). nuc ish 16p13.11(NDE1-MYH11x3).

Discordant to the aCGH result, metaphase FISH analysis in the mother showed a deletion pattern, with only one probe signal on one chromosome 16 . This signal was also brighter than usual, suggesting that two copies of 16p13.11 were present on that chromosome (Figure 1e). Further interphase nuclei FISH testing was then performed, and two signals adjacent to one another were observed (Figure 1f). Therefore, in combination with the aCGH result, FISH findings were interpreted as a balanced interchromosomal rearrangement at 16p12.3-p13.11, resulting in one chromosome 16 with an interstitial deletion and the other with an interstitial insertion. The karyotype of the mother was described as follows: ish $\operatorname{der}(16)$ ins $(16)(16 ; 16)$ (p1?2.3;p1?2.3p1?3.11)(NDE1-MYH11enh),der(16)ins(16) (16;16)(p1?2.3;p1?2.3p1?3.11)(NDE1-MYH11-). nuc ish 16p13.11(NDE1-MYH11x2).
Interpretation of the rearrangement in the mother and its associated recurrence risk focused attention on the proband's elder brother. By means of MLPA, the same results as in the proband were obtained, indicating that both children had inherited the derivative maternal chromosome 16 carrying two copies of 16p13.11. Thus, their karyotypes were described as: ish $\operatorname{der}(16)$ ins(16) (16;16)(p1?2.3;p1?2.3p1?3.11)(NDE1-MYH11enh)mat.

Surprisingly, further familial studies showed that both the maternal grandfather and the maternal uncle also carried the same duplication as the proband.

The analysis of three STR markers mapping within the region delimited by aCGH supported previous gene dosage results in the children, maternal grandfather, and maternal uncle. In the mother, the grandpaternal genotype was observed in two of the three markers. However, all other informative markers (6) in chromosome 16 showed biparental inheritance. The same results were obtained in DNA from buccal swab in the mother, and they are consistent with a deletion of the maternal allele of 16p13.11 (Figure 2). 


\section{Discussion}

Only a few cases describing homologous balanced rearrangements have been reported so far. Most of them occurred at 22q11.2 region [1-3], which is well known for being rich in low copy repeats (LCRs). Most constitutional translocations involving $22 \mathrm{q} 11$ share the same $22 \mathrm{q} 11.2$ breakpoint located within LCR-B, and these breakpoints are usually located at the center of palindromic AT-rich repeat sequences (PATRRs) [10]. Therefore, palindromemediated translocations have been suggested as one of the mechanisms for human chromosomal rearrangements. The short arm of chromosome 16 has a similar structure with over $10 \%$ of the euchromatic region being composed of LCRs which promote non-allelic homologous recombination (NAHR) $[7,11,12]$.
Haplotype analysis suggests two possible mechanisms underlying the origin of the balanced rearrangement in the proband's mother. We can hypothesize that previous to conception, a meiotic NAHR might have occurred in the proband's grandmother (we also cannot exclude the possibility of a germline mosaicism) with the subsequent transmission to the proband's mother of the deleted chromosome 16. A duplicated chromosome 16 would have been transmitted by the proband's grandfather. A second and more likely mechanism is a mitotic NAHR between a maternal and paternal chromatid within the first zygotic divisions that would originate four chromatids: the two recombination products (one with a triplication and the other one with the reciprocal deletion) and two original parental chromatids, carrying a duplication and a normal 


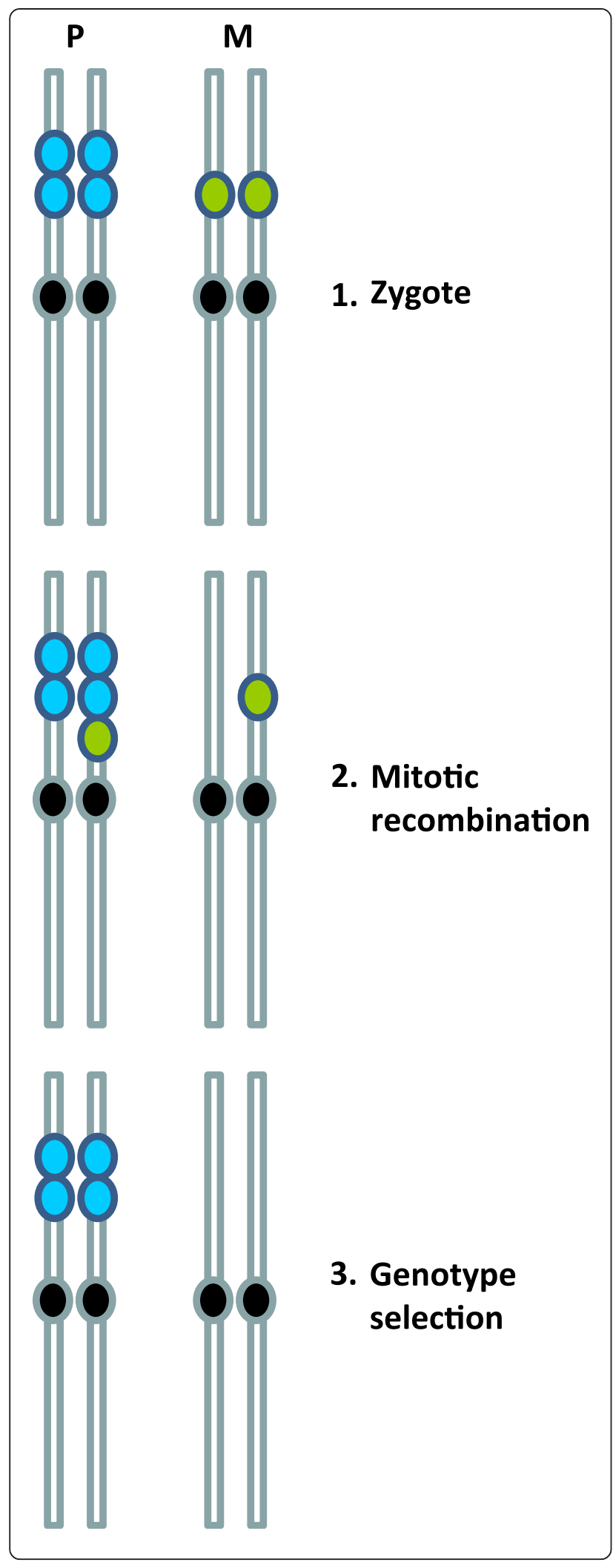

Figure 3 Postzygotic hypothesis for the rearrangement in chromosome 16 leading to genomic compensation in the mother (II:3). Mitotic recombination between a paternal chromatid carrying the duplication and a normal maternal chromatid during the first zygotic divisions would originate four different chromatids, two of which would be selected in a compensated genotype. Circles indicate region 16p13.11, which is colored as in Figure 2. P and M designate the paternal and maternal chromosome, respectively.

dosage. This event would be followed by selection of cells carrying the two compensated non-sister chromatids in the embryo, in a model similar to the one proposed by Carelle-Calmels et al. [1] (Figure 3).

A consequence of postzygotic NAHR is chromosomal mosaicism. Studies in the mother revealed the same genotype in buccal swab and peripheral blood lymphocytes, suggesting that cell lineages with different genotypes might have been restricted to other tissues or would have been confined to extraembryonic tissues, a likely explanation if the NAHR occurred in fact early after conception.

LCR-mediated NAHR may originate unstable products that are more prone to act as substrates of new rearrangements in further generations [4]. In this case, it is noteworthy that, considering the meiotic recombinations observed in the grandparental gametes, both the proband's mother and maternal uncle received the same parental genotypic contribution in $16 \mathrm{p} 13.11$, but the proposed rearrangement occurred only in the mother (Figure 2). This rearrangement resulted in gene dosage compensation, preventing the possible phenotypic effects of the CNV in that woman.

The phenotype of patients with 16p13.11 duplication is very variable and it has been associated with clinical features including behavioral abnormalities, autistic spectrum disorders, congenital heart defects, skeletal manifestations, and developmental delay [8]. Significant association has been described for schizophrenia and intellectual disability [13], and brain malformations have been sporadically observed [14]. Psychomotor development in the proband and his brother was normal at the time of the assessment, although future cognitive handicap cannot be ruled out. NDE1, one of the genes included in the duplicated segment, is strongly expressed in brain, and it forms complexes with LIS1, a dosage-sensitive gene crucial for neuronal migration and cerebral development [15] and known to underlie Miller-Dieker lissencephaly syndrome (MIM 247200) [5]. Notwithstanding, since the $\mathrm{CNV}$ is also present in other healthy family members, we cannot associate it to the cerebral malformations observed in the proband so it still remains as a finding of uncertain significance.

On the other hand, this uncommon rearrangement in chromosome 16 in the mother of the proband raises the risk of an unbalanced pregnancy to $100 \%$, since all of 
her offspring will either inherit the 16p13.11 duplication or the deletion. The detection of this unusual rearrangement emphasizes the need of parental FISH studies in order to be able to offer accurate genetic counseling for future pregnancies.

\section{Conclusions}

The increasing detection of these unusual rearrangements reinforces the need to determine the genomic location of interstitial gains or losses detected by aCGH [16]. Consequently, combined in situ hybridization and genomic parental approaches are crucial in order to rule out any balanced parental rearrangement that may involve a very high recurrence risk.

\section{Abbreviations}

aCGH: Array-based comparative genomic hybridization; CNV: Copy number variation; FISH: Fluorescence in situ hybridization; IT: Insertional translocation; LCR: Low copy repeat; MLPA: Multiplex ligation-dependent probe amplification; MRI: Magnetic resonance imaging; NAHR: Non-allelic homologous recombination; PATRR: Palindromic AT-rich repeat; STR: Short tandem repeat.

\section{Competing interests}

The authors declare that they have no competing interests.

\section{Authors' contributions}

AD conceived the study and wrote the manuscript. LF carried out the STR markers segregation studies and co-wrote the manuscript. MT carried out the cytogenetic and FISH studies. JN carried out the MLPA studies and participated in the draft of the manuscript. FGS participated in the draft of the manuscript. RR carried out the prenatal clinical evaluation. EM participated in the draft of the manuscript. MP and EV participated in the aCGH analysis. FSS carried out the clinical evaluation of the family. MM carried out the cytogenetic and aCGH studies. PL participated in the draft of the manuscript. All authors read and approved the final manuscript.

\section{Acknowledgements}

We thank all technical staff of the Cytogenetics and Genomics Units. We are very grateful to Dr. Jean McGowan-Jordan for his suggestions about cytogenetic nomenclature. FISH and aCGH materials were supported by grant 08/PI1207 from Fondo de Investigaciones Sanitarias (FIS) and research project ENDOSCREEN (S2011/BMD-2396) from Comunidad de Madrid.

\section{Author details}

${ }^{1}$ Instituto de Genética Médica y Molecular (INGEMM), IdiPAZ, Hospital Universitario La Paz, Paseo de la Castellana 261, 28046 Madrid, Spain. ${ }^{2}$ CIBER de Enfermedades Raras (CIBERER), ISCIII, Madrid, Spain. ${ }^{3}$ Servicio de Fisiopatología Fetal, Hospital Universitario La Paz, Madrid, Spain.

Received: 20 December 2013 Accepted: 3 October 2014 Published online: 29 October 2014

\section{References}

1. Carelle-Calmels N, Saugier-Veber P, Girard-Lemaire F, Rudolf G, Doray B, Guérin E, Kuhn P, Arrivé M, Gilch C, Schmitt E, Fehrenbach S, Schnebelen A, Frébourg T, Flori E: Genetic compensation in a human genomic disorder. N Engl J Med 2009, 360(12):1211-1216.

2. Alkalay AA, Guo T, Montagna C, Digilio MC, Dallapiccola B, Marino B, Morrow B: Genetic dosage compensation in a family with velo-cardio-facial/DiGeorge/ 22q11.2 deletion syndrome. Am J Med Genet A 2011, 155A(3):548-554.

3. Fernández L, Nevado J, De Torres ML, Mansilla E, Vallespín E, García-Miñaúr S, Palomo R, Deirós L, Cabrera M, Galo ED, Lapunzina P, Delicado A: Additional case of an uncommon 22q11.2 reciprocal rearrangement in a phenotypically normal mother of children with 22q11.2 deletion and 22q11.2 duplication syndromes. Am J Med Genet A 2012, 158A(11):2963-2968
4. Nowakowska BA, de Leeuw N, Ruivenkamp CA, Sikkema-Raddatz B, Crolla $J A$, Thoelen R, Koopmans M, den Hollander N, van Haeringen A, van der Kevie-Kersemaekers AM, Pfundt R, Mieloo H, van Essen T, de Vries BB, Green A, Reardon W, Fryns JP, Vermeesch JR: Parental insertional balanced translocations are an important cause of apparently de novo CNVs in patients with developmental anomalies. Eur J Hum Genet 2012, 20(2):166-170.

5. Hannes FD, Sharp AJ, Mefford HC, de Ravel T, Ruivenkamp CA, Breuning $\mathrm{MH}$, Fryns JP, Devriendt K, Van Buggenhout G, Vogels A, Stewart $H_{\text {, }}$ Hennekam RC, Cooper GM, Regan R, Knight SJ, Eichler EE, Vermeesch JR: Recurrent reciprocal deletions and duplications of 16p13.11: the deletion is a risk factor for MR/MCA while the duplication may be a rare benign variant. J Med Genet 2009, 46(4):223-232.

6. Ullmann R, Turner G, Kirchhoff M, Chen W, Tonge B, Rosenberg C, Field M, Vianna-Morgante AM, Christie L, Krepischi-Santos AC, Banna L, Brereton AV, Hill A, Bisgaard AM, Müller I, Hultschig C, Erdogan F, Wieczorek G, Ropers $\mathrm{HH}$ : Array CGH identifies reciprocal 16p13.1 duplications and deletions that predispose to autism and/or mental retardation. Hum Mutat 2007, 28(7):674-682.

7. Nagamani SC, Erez A, Bader P, Lalani SR, Scott DA, Scaglia F, Plon SE, Tsai CH, Reimschisel T, Roeder E, Malphrus AD, Eng PA, Hixson PM, Kang SH, Stankiewicz P, Patel A, Cheung SW: Phenotypic manifestations of copy number variation in chromosome 16p13.11. Eur J Hum Genet 2011, 19(3):280-286.

8. Ramalingam A, Zhou XG, Fiedler SD, Brawner SJ, Joyce JM, Liu HY, Yu S: 16 p13.11 duplication is a risk factor for a wide spectrum of neuropsychiatric disorders. J Hum Genet 2011, 56(7):541-544.

9. Vallespín E, Palomares Bralo M, Mori M, Martín R, García-Miñaúr S, Fernández L, de Torres ML, García-Santiago F, Mansilla E, Santos F, M-Montaño VE, Crespo MC, Martín S, Martínez-Glez V, Delicado A, Lapunzina P, Nevado J: Customized high resolution $\mathrm{CGH}$-array for clinical diagnosis reveals additional genomic imbalances in previous well-defined pathological samples. Am J Med Genet A 2013, 161A(8):1950-1960.

10. Kato T, Kurahashi H, Emanuel BS: Chromosomal translocations and palindromic AT-rich repeats. Curr Opin Genet Dev 2012, 22(3):221-228.

11. Loftus BJ, Kim UJ, Sneddon VP, Kalush F, Brandon R, Fuhrmann J, Mason T, Crosby ML, Barnstead M, Cronin L, Deslattes Mays A, Cao Y, Xu RX, Kang HL, Mitchell S, Eichler EE, Harris PC, Venter JC, Adams MD: Genome duplications and other features in $12 \mathrm{Mb}$ of DNA sequence from human chromosome 16p and 16q. Genomics 1999, 60(3):295-308.

12. Martin J, Han C, Gordon LA, Terry A, Prabhakar S, She X, Xie G, Hellsten U, Chan YM, Altherr M, Couronne O, Aerts A, Bajorek E, Black S, Blumer H, Branscomb E, Brown NC, Bruno WJ, Buckingham JM, Callen DF, Campbell CS, Campbell ML, Campbell EW, Caoile C, Challacombe JF, Chasteen LA, Chertkov O, Chi HC, Christensen M, Clark LM, et al: The sequence and analysis of duplication-rich human chromosome 16. Nature 2004, 432(7020):988-994.

13. Mefford HC, Cooper GM, Zerr T, Smith JD, Baker C, Shafer N, Thorland EC, Skinner C, Schwartz CE, Nickerson DA, Eichler EE: A method for rapid, targeted CNV genotyping identifies rare variants associated with neurocognitive disease. Genome Res 2009, 19(9):1579-1585.

14. Kariminejad R, Lind-Thomsen A, Tümer Z, Erdogan F, Ropers HH, Tommerup $\mathrm{N}$, Ullmann R, Møller RS: High frequency of rare copy number variants affecting functionally related genes in patients with structural brain malformations. Hum Mutat 2011, 32(12):1427-1435.

15. Feng $Y$, Walsh CA: Mitotic spindle regulation by Nde1 controls cerebral cortical size. Neuron 2004, 44(2):279-293.

16. Neill NJ, Ballif BC, Lamb AN, Parikh S, Ravnan JB, Schultz RA, Torchia BS, Rosenfeld JA, Shaffer LG: Recurrence, submicroscopic complexity, and potential clinical relevance of copy gains detected by array CGH that are shown to be unbalanced insertions by FISH. Genome Res 2011, 21(4):535-544.

\section{doi:10.1186/s12881-014-0116-3}

Cite this article as: Delicado et al: Familial imbalance in 16p13.11 leads to a dosage compensation rearrangement in an unaffected carrier. BMC Medical Genetics 2014 15:116. 